\title{
Reporting of academic degrees in high-impact medical
}

\section{journals [version 1; peer review: 1 approved with reservations,}

\section{1 not approved]}

\author{
Nikola Stankovic¹, Jerry P. Nolan², Lars W. Andersen (iD)1 \\ ${ }^{1}$ Research Center for Emergency Medicine, Department of Clinical Medicine, Aarhus University and Aarhus University Hospital, \\ Aarhus, Denmark \\ ${ }^{2}$ Royal United Hospital, Bath, UK
}

\section{V1 First published: 05 Nov 2019, 8:1852 \\ https://doi.org/10.12688/f1000research.21096.1 \\ Second version: 12 Dec 2019, 8:1852 \\ https://doi.org/10.12688/f1000research.21096.2 \\ Latest published: 20 Feb 2020, 8:1852 \\ https://doi.org/10.12688/f1000research.21096.3}

\section{Abstract}

Academic degrees following author names are often included in medical research papers. However, it remains unclear how many journals choose to include academic degrees and whether this is more common in certain types of journals. We examined the 100 highest impact medical journals and found that only 24 medical journals reported academic degrees. Moreover, this was substantially more common in journals based in North America compared with Europe. Further research is required to explore the implications of listing academic degrees on the readers' attitude towards research quality.

\section{Keywords}

Academic, degree, impact factor, journals, publication, high-impact

This article is included in the Research on

Research, Policy \& Culture gateway.

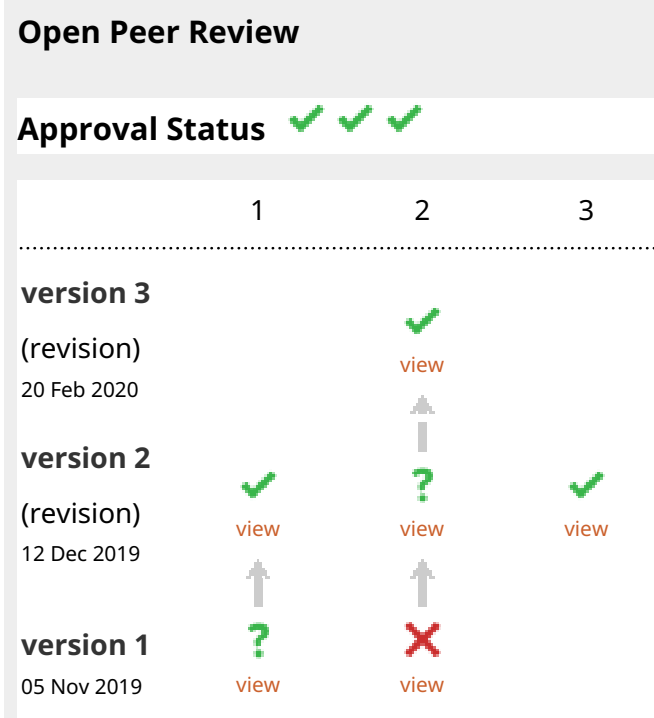

1. Saif Aldeen AIRyalat , The University of Jordan, Amman, Jordan

2. Ana Marusic (D), University of Split, Split, Croatia

3. Christopher C. Winchester (ID), University of Split, Split, Croatia

Any reports and responses or comments on the article can be found at the end of the article. 
Corresponding author: Lars W. Andersen (Iwandersen@clin.au.dk)

Author roles: Stankovic N: Data Curation, Formal Analysis, Investigation, Methodology, Project Administration, Validation, Writing Original Draft Preparation, Writing - Review \& Editing; Nolan JP: Conceptualization, Methodology, Writing - Review \& Editing; Andersen LW: Conceptualization, Data Curation, Formal Analysis, Investigation, Methodology, Project Administration, Supervision, Validation, Writing - Original Draft Preparation, Writing - Review \& Editing

Competing interests: Jerry Nolan is the editor-in-chief for Resuscitation. There are no other competing interests.

Grant information: The author(s) declared that no grants were involved in supporting this work.

Copyright: @ 2019 Stankovic $\mathrm{N}$ et al. This is an open access article distributed under the terms of the Creative Commons Attribution License, which permits unrestricted use, distribution, and reproduction in any medium, provided the original work is properly cited.

How to cite this article: Stankovic N, Nolan JP and Andersen LW. Reporting of academic degrees in high-impact medical journals [version 1; peer review: 1 approved with reservations, 1 not approved] F1000Research 2019, 8:1852

https://doi.org/10.12688/f1000research.21096.1

First published: 05 Nov 2019, 8:1852 https://doi.org/10.12688/f1000research.21096.1 


\section{Introduction}

During submission of research papers to medical journals, authors are often asked to include academic degrees, affiliations, and/or job titles. However, journals differ in how they present this information to readers. While some journals include academic degrees following author names, others choose not to list this information on the title page. It is unclear how many journals choose to include academic degrees and whether this is more common in certain types of journals. Among the most influential medical journals, we examined journal factors associated with the inclusion of academic degrees on the title page.

\section{Methods}

We identified the hundred highest impact medical journals based on impact factor reported in the Journal Citation Reports ${ }^{1}$ published in 2018. Characteristics of each journal in regard to specialty, impact factor, primary journal focus, continent, and open access policy were obtained. Data were collected on the presence of academic degrees following author names in the title page by assessment of multiple original research articles from each journal. Approximately ten articles published in July 2018 and August 2019 were assessed for each journal. If there was any discrepancy between the print and the online version, the print version was used. There were no discrepancies within journals for the two time periods.

Descriptive statistics were used to characterize the journals. Categorical data were compared with the Fisher's Exact Test and continuous data were compared with the Wilcoxon

${ }^{1}$ https://jcr.clarivate.com/JCRLandingPageAction.action Accessed September $7^{\text {th }} 2019$.
Rank-Sum Test. The association between journal characteristics and the reporting of academic degrees were estimated using multivariable logistic regression.

Statistical analyses were performed in SAS (version 9.4). A two-tailed $\mathrm{p}<0.05$ was considered significant.

\section{Results}

Of the 100 highest impact medical journals, 24 journals reported academic degrees on the title page (Table 1). We found that $49 \%$ of journals were published in Europe and $51 \%$ were published in North America. Only 8\% of European journals reported academic degrees while $39 \%$ of North American journals reported academic degrees. The median impact factor of journals reporting and not reporting academic degrees was 12 (IQR 11-20) and 15 (IQR $10-19$ ), respectfully.

Multivariable analysis showed that North America and a clinical journal focus was associated with increased odds of reporting academic degrees (Table 1). No association was found for the other journal characteristics.

\section{Discussion}

Among the 100 highest impact medical journals, only 24 journals reported academic degrees following author names on the title page. Reporting of academic degrees was substantially more common in journals based in North America compared with Europe.

Listing author academic degrees is an editorial policy decision but there is little guidance from the International Committee of Medical Journals Editors (ICMJE) or the American Medical Association (AMA) Manual of Style. Specifically,

Table 1. The presence of academic degree(s) according to journal factors in the top 100 high-impact medical journals.

\begin{tabular}{|c|c|c|c|c|c|}
\hline & & \multicolumn{2}{|c|}{$\begin{array}{l}\text { Presence of academic } \\
\text { degree }\end{array}$} & \multicolumn{2}{|c|}{$\begin{array}{l}\text { Multivariable } \\
\text { model }\end{array}$} \\
\hline & & Yes $(n=24)$ & No $(n=76)$ & OR & $95 \% \mathrm{Cl}$ \\
\hline \multirow{3}{*}{ Primary journal focus } & Clinical & $17(71 \%)$ & $26(34 \%)$ & Ref. & - \\
\hline & Basic science and/or experimental & $0(0 \%)$ & $21(28 \%)$ & $\mathrm{NA}^{\mathrm{a}}$ & $\mathrm{NA}^{\mathrm{a}}$ \\
\hline & Combined & $7(29 \%)$ & $29(38 \%)$ & 0.25 & $0.07-0.92$ \\
\hline \multirow{2}{*}{ Specialty } & General & $6(25 \%)$ & $12(16 \%)$ & Ref. & - \\
\hline & Specific & $18(75 \%)$ & $64(84 \%)$ & 1.05 & $0.23-4.93$ \\
\hline \multirow{3}{*}{ Open access } & Yes & $2(8 \%)$ & $8(11 \%)$ & Ref. & - \\
\hline & Partly & $15(63 \%)$ & $24(32 \%)$ & 2.31 & $0.22-23.9$ \\
\hline & No & 7 (29\%) & $44(58 \%)$ & 0.90 & $0.09-9.21$ \\
\hline \multirow{2}{*}{ Continent } & Europe & $4(17 \%)$ & $45(59 \%)$ & Ref. & - \\
\hline & North America & $20(83 \%)$ & $31(41 \%)$ & 9.57 & $2.38-38.4$ \\
\hline Journal impact & Impact factor & $12(10-19)$ & $15(11-20)$ & 1.00 & $0.98-1.02$ \\
\hline
\end{tabular}

Not able to be estimated as no journal in this category reported academic degrees

Median with quartiles 
the ICMJE states "Each author's highest academic degrees should be listed, although some journals do not publish these $e^{2}$." and the AMA Manual of Style writes "Journals should establish their own policies on the inclusion of authors' degrees." . Neither provides a rationale for providing academic degrees and it remains unclear why some journals do and others do not. The marked difference between journals published in North America compared with Europe cannot be explained by the current study but may be a reflection of cultural differences in attitude towards degrees and titles. Further research is needed to explore the implications of listing academic degrees on the readers' attitude towards research quality.

Limitations of the current study include that we only evaluated high-impact medical journals. Furthermore, we only assessed some journal characteristics. We evaluated only recent issues of these journals and are therefore unable to comment on trends in the use of author academic degrees.

${ }^{2}$ http://www.icmje.org/recommendations/browse/manuscript-preparation/ preparing-for-submission.html\#a

${ }^{3}$ https://www.amamanualofstyle.com/view/10.1093/jama/9780195176339. 001.0001/med-9780195176339-div2-10
In conclusion, we found that academic degrees are reported in about one fourth of medical journals and that this practice is more common in North America.

\section{Data availability}

Underlying data

Harvard Dataverse: Replication Data for: Reporting of academic degrees in high-impact medical journals, https://doi. org/10.7910/DVN/KTWS6C ${ }^{4}$

This project contains the following underlying data:

- CSV file with the titles of the medical journals investigated

Data are available under the terms of the Creative Commons Zero "No rights reserved" data waiver (CC0 1.0 Public domain dedication).

The Journal Citation Report from Clarivate Analytics can only be accessed through an individual or institutional account.

${ }^{4}$ Stankovic, Nikola, 2019, "Replication Data for: Reporting of academic degrees in high-impact medical journals", https://doi.org/10.7910/DVN/ KTWS6C, Harvard Dataverse, V1 


\section{Open Peer Review}

\section{Current Peer Review Status: ? X}

\section{Version 1}

Reviewer Report 18 November 2019

https://doi.org/10.5256/f1000research.23217.r56491

(C) 2019 Marusic A. This is an open access peer review report distributed under the terms of the Creative Commons Attribution License, which permits unrestricted use, distribution, and reproduction in any medium, provided the original work is properly cited.

\section{Ana Marusic}

Department of Research in Biomedicine and Health, School of Medicine, University of Split, Split, Croatia

This article addresses an interesting and intriguing question in publishing - why author's academic degrees are published in journals. Editorial guidelines mention this, but do not provide actual guidance.

This research showed that journals from North America and from clinical disciplines are more likely to publish the degrees, which is an interesting finding, at least in high-impact journals. The manuscript is written clearly, as a brief report, and follows a logical order, with appropriate statistical analysis.

I have two major issues:

1. There is no literature overview on this problem (or a statement that there is no evidence on this issue in literature).

2. The description of the selection of articles in the set of 100 journals is vague. It is not clear what was the method for sampling "approximately ten articles ..." in the two study years. This needs to be clarified.

Is the work clearly and accurately presented and does it cite the current literature? Partly

Is the study design appropriate and is the work technically sound? Partly

Are sufficient details of methods and analysis provided to allow replication by others? Partly

If applicable, is the statistical analysis and its interpretation appropriate? Yes 
Are all the source data underlying the results available to ensure full reproducibility? Yes

Are the conclusions drawn adequately supported by the results?

Yes

Competing Interests: No competing interests were disclosed.

Reviewer Expertise: Journal and peer review research

I confirm that I have read this submission and believe that I have an appropriate level of expertise to state that I do not consider it to be of an acceptable scientific standard, for reasons outlined above.

Author Response 06 Dec 2019

Lars Andersen

Comment 1:

"There is no literature overview on this problem (or a statement that there is no evidence on this issue in literature)."

\section{Response 1:}

Thank you for raising an important point in the manuscript. To our knowledge following literature review - there are no studies evaluating the impact of reporting academic degrees following author names in medical journals. We agree that a statement addressing that no evidence on this issue is available, will add valuable content to the manuscript. It should be noted, however, that the current study does not evaluate why journals report academic degrees or whether it has an impact on the readers. In this study, we evaluated whether high-impact medical journals reported academic degrees and whether certain journal factors were associated with reporting academic degrees. Further research is needed to evaluate the impact of reporting academic degrees on readers' perception of research.

The following was rephrased in the Introduction section: "To our knowledge, no evidence is available on how many journals choose to include academic degrees and whether this is more common in certain types of journals."

We chose not to include a statement addressing that no evidence is available on the impact of reporting academic degrees following author names in medical journals in the Introduction section, since it may be out of scope with our aim of the current study. However, an appropriate statement has been provided in the Discussion section: "To our knowledge, no studies exist on the impact of reporting academic degrees in medical journals, why further research is needed to explore the implications of listing academic degrees on the readers' attitude towards research quality."

\section{Comment 2:}

"The description of the selection of articles in the set of 100 journals is vague. It is not clear what was the method for sampling "approximately ten articles ..." in the two study years. This needs to be clarified." 


\section{Response 2:}

We agree that this definition needs to be clarified. We evaluated numerous original research articles in multiple journal issues to assess any discrepancy in reporting of academic degrees over the time period from July 2018 to August 2019. This would account to approximately 10 research articles. No discrepancy in reporting academic degrees during this time period within each journal was identified.

Following was rephrased to clarify this issue in the Methods section: "Multiple articles published throughout July 2018 and August 2019 were assessed for each journal. No discrepancy of reporting academic degrees throughout the time period was identified."

Competing Interests: No competing interests were disclosed.

Reviewer Report 08 November 2019

\section{https://doi.org/10.5256/f1000research.23217.r56195}

(c) 2019 AlRyalat S. This is an open access peer review report distributed under the terms of the Creative Commons Attribution License, which permits unrestricted use, distribution, and reproduction in any medium, provided the original work is properly cited.

\section{Saif Aldeen AlRyalat}

Department of Ophthalmology, University of Jordan Hospital, The University of Jordan, Amman, Jordan

Stankovic et al assessed the factors associated with mentioning academic degrees on the journal's title page. The current study is interesting and sheds a light on an important topic, although the authors chose a restricted sample to analyze (i.e. high impact medical journals). I believe that the current report can be improved by clarifying some points within its methods section, as follows.

In the methods section, the authors did not define "academic degrees". In the data associated with this study, they included columns on "Academic degrees", "job titles", and "fellowship designations". In medicine, reporting of fellowships might be more important than the academic degree, especially if the researcher is a clinician working in a non-academic institution. The authors should clearly define what they mean by "academic degree" that they used in their analysis.

In the methods section, the authors categorized journals according to specialty as general or specific. The authors should detail the basis of this classification. Moreover, the authors categorized open access status into yes, no, or partly, where "partly" is not a common word used to describe the open access status (examples of common, well-defined words include green open access, hybrid access and so on). By going back to the data, I observed several non-open access journals categorized as "partly", so I would suggest adopting strict criteria for categorization, such as the one suggested by Scopus: 
"Open Access Journals are indicated as Open Access if the journal is listed in the Directory of Open Access Journals (DOAJ) and/or the Directory of Open Access Scholarly Resources (ROAD)".

In the discussion section, the authors should include studies that discussed the impact of an academic degree in publishing, which would complement the points raised by the authors in the present report.

Is the work clearly and accurately presented and does it cite the current literature? Partly

Is the study design appropriate and is the work technically sound?

Yes

Are sufficient details of methods and analysis provided to allow replication by others? Partly

If applicable, is the statistical analysis and its interpretation appropriate? Yes

Are all the source data underlying the results available to ensure full reproducibility? Yes

Are the conclusions drawn adequately supported by the results? Yes

Competing Interests: No competing interests were disclosed.

Reviewer Expertise: Medicine, bibliometrics, ophthalmology.

I confirm that I have read this submission and believe that I have an appropriate level of expertise to confirm that it is of an acceptable scientific standard, however I have significant reservations, as outlined above.

Author Response 06 Dec 2019

Lars Andersen

Comment 1:

"In the methods section, the authors did not define "academic degrees". In the data associated with this study, they included columns on "Academic degrees", "job titles", and "fellowship designations". In medicine, reporting of fellowships might be more important than the academic degree, especially if the researcher is a clinician working in a non-academic institution. The authors should clearly define what they mean by "academic degree" that they used in their analysis."

Response 1:

Thank you for this comment, which we agree will add clarification on the definition of 
"academic degrees". Since "fellowship designations" were reported in six medical journals only - which all included academic degrees - we decided not to include a separate analysis for "fellowship designations". Job titles were present in one medical journal, and this was available online only, why we chose to exclude this category from the final analysis.

Thus, we included following clarification on the definition of academic degrees in the methods section of the manuscript: "The presence of academic degrees was defined as the inclusion of academic degree abbreviations following author names (e.g. MD, PhD, RN, MPH etc.)."

\section{Comment 2:}

"In the methods section, the authors categorized journals according to specialty as general or specific. The authors should detail the basis of this classification.

\section{Response 2:}

Agree. The medical journals were categorized as "general", "specific" or "surgical" based on the specialty heterogeneity reported in the journal. Journals with high heterogeneity of reported specialties were categorized as "general". In the statistical analysis, "surgical" was grouped with "specific", since only two journals were categorized as "surgical".

However, to avoid equivocality, we have redefined the basis of our specialty classification for the journals. The journals are now classified according to subject area designation in Scopus. Consequently, the results have been adjusted according to this classification.

Thus, we included following phrase in the methods section: "Journals were categorized as "general" or "specific" based on subject area designation in Scopus", i.e. whether content was primarily related to one specialty (e.g. cardiology) or multiple specialties."

\section{Comment 3}

"Moreover, the authors categorized open access status into yes, no, or partly, where "partly" is not a common word used to describe the open access status (examples of common, well-defined words include green open access, hybrid access and so on). By going back to the data, I observed several non-open access journals categorized as "partly", so I would suggest adopting strict criteria for categorization, such as the one suggested by Scopus: "Open Access Journals are indicated as Open Access if the journal is listed in the Directory of Open Access Journals (DOAJ) and/or the Directory of Open Access Scholarly Resources (ROAD)"."

\section{Response 3:}

Thank you for this insightful suggestion. We agree with the need to adopt strict criteria for categorization (such as Scopus). We have adopted Scopus' definition of open access. The journals within the dataset have been reassessed according to Scopus' definition of open access, and the current dataset, results and manuscript reflect the changes accordingly.

Consequently, we have included following phrase in the methods section: "Open access was identified based on open access designation in Scopus ${ }^{2}$. Scopus registers open 
access as follows: "In Scopus, journals are registered as being OA journals only if they are registered as Gold OA or Subsidized OA at one or both of the following sources: Directory of Open Access Journals (DOAJ) and the Directory of Open Access Scholarly Resources $(R O A D)^{\prime \prime 3}$."

\section{Comment 4:}

"In the discussion section, the authors should include studies that discussed the impact of an academic degree in publishing, which would complement the points raised by the authors in the present report."

\section{Response 4:}

Thank you for this comment. We agree that it would be valuable to include studies discussing the impact of an academic degree in publishing. However, to our knowledge, no studies have evaluated the impact of reporting academic degrees in journals, why it was not possible to include this in the discussion section.

A statement has been provided in the Discussion section to address the lack of studies examining the impact of reporting academic degrees in publishing: "To our knowledge, no studies exist on the impact of reporting academic degrees in medical journals, why further research is needed to explore the implications of listing academic degrees on the readers' attitude towards research quality."

Competing Interests: No competing interests were disclosed.

The benefits of publishing with F1000Research:

- Your article is published within days, with no editorial bias

- You can publish traditional articles, null/negative results, case reports, data notes and more

- The peer review process is transparent and collaborative

- Your article is indexed in PubMed after passing peer review

- Dedicated customer support at every stage

For pre-submission enquiries, contact research@f1000.com 